\title{
Padma 28 - eine sinnvolle Ergänzung zur Behandlung der peripheren arteriellen Verschlusskrankheit im Stadium Ila und Ilb
}

\section{Christian Regli Ernst Groechenig}

Angiologie, Kantonsspital Aarau, Schweiz

\section{Schlüsselwörter}

PAVK - Schaufensterkrankheit · Fontaine $\cdot A B I$. Padma 28

\section{Zusammenfassung}

Die periphere arterielle Verschlusskrankheit (PAVK) ist zu über $90 \%$ durch Atherosklerose bedingt. Da diese alle Arterien des Körpers betreffen kann, gilt die PAVK als wichtiger Indikator für kardiovaskuläre Ereignisse wie Herzinfarkt oder Schlaganfall und somit für die häufigste Todesursache in der westlichen Welt. Mit der ABI-Messung (Knöchel/Arm-Druck-Messung) kann die Krankheit auf eine im Praxisalltag gut durchführbare Weise diagnostiziert werden. Die Grundlage der Behandlung der Atherosklerose beinhaltet neben der Lifestyle-Änderung eine medikamentöse Behandlung der bekannten Risikofaktoren. Liegt eine schwere Durchblutungsstörung mit kritischer Ischämie vor, ist eine kathetertechnische oder chirurgische Revaskularisation zwingend. Von einer Schaufensterkrankheit sprechen wir, wenn aufgrund der PAVK die Gehstrecke limitiert ist. Hier kann neben interventionellen Behandlungen (perkutane transluminale Angioplastie (PTA) / Chirurgie) auch ein konservatives Gehtraining durchgeführt werden. Der supportive Einsatz von Padma 28 bei der konservativen Behandlung zeigt einen messbaren Effekt mit einer deutlichen Verlängerung der maximalen Gehstrecke und ist als additive Therapie eine zu prüfende Behandlungsoption.
Hinweis: Padma 28 (Swissmedic No. 58436) ist in der Schweiz auch unter den Namen Padmed Circosan (Swissmedic No. 60131) und Arteria-vita (Swissmedic No. 62863) erhältlich und kassenzulässig.

\section{Keywords}

PAOD - Intermittent claudication - Fontaine $\cdot A B I$. Padma 28

\section{Summary}

Padma 28 - a Useful Supplement in the Treatment of Peripheral Arterial Occlusive Disease in the Stages Ila and IIb

Peripheral arterial occlusive disease (PAOD) is in about $90 \%$ of the cases caused by atherosclerosis. Since this can affect all arteries of the body, PAOD is considered an important indicator of cardiovascular events such as heart attack or stroke, and thus of the leading cause of death in the Western world. With the measurement of the ankle/brachial pressure index $(A B I)$, a practicable method is available to diagnose the disease in routine practice. Besides lifestyle modifications, the mainstay of treatment of atherosclerosis includes also drug treatment of known risk factors. In case of a severe circulatory disorder with critical ischemia, a revascularization by angioplasty or surgical methods is imperative. If the walking distance is limited due to PAOD, we speak of intermittent claudication. Here, in addition to the interventional treatment (percutaneous transluminal angioplasty (PTA) / surgery), a conservative walking exercise can be performed. The supportive use of Padma 28 in the conservative treatment has a measurable effect with a significant extension of the maximum walking distance and poses an additive treatment option. 
Die periphere arterielle Verschlusskrankheit (PAVK) ist eine Manifestation der Atherosklerose der Bein- und Armarterien, einer sehr ernst zu nehmenden Erkrankung. Im Hinblick auf die Prognose ist sie vergleichbar mit dem Kolonkarzinom und stellt ein erhebliches Risiko für kardiovaskuläre Ereignisse dar. Da es sich um ein und dieselbe Erkrankung mit unterschiedlicher Lokalisation handelt, sprechen wir von peripherer, koronarer, zerebraler oder viszeraler arterieller Verschlusskrankheit. Die kardiovaskuläre Erkrankung mit Folgen wie Herzinfarkt, Schlaganfall oder Amputation von Extremitäten ist die häufigste Todesursache in der westlichen Welt. Die PAVK ist dafür ein bislang weit unterschätzter Risikofaktor.

Nur ein kleiner Teil der Betroffenen bemerkt PAVK-Symptome [1]. Diese äußern sich als schmerzbedingte Gehstreckeneinschränkung mit Beinschmerzen (Schaufenstererkrankung), die sich nach Beendigung der Belastung rasch wieder bessern. Die PAVK ist mit weit mehr verbunden als mit einer Einschränkung der Gehfähigkeit oder einem erhöhten Amputationsrisiko. Zur Verminderung des Risikos gefürchteter kardiovaskulärer Ereignisse müssen Patienten mit einer PAVK erkannt und behandelt werden.

Die ABI-Messung (Knöchel/Arm-Druck-Messung) ist eine im Praxisalltag gut durchführbare Methode zur Feststellung einer PAVK. Mit Doppler-Ultraschall wird der Arteriendruck am Knöchel und am Arm gemessen, der normalerweise gleich hoch ist (Quotient 1). Liegt eine Verengung der Arterien oder gar ein Verschluss vor, ist der Knöcheldruck niedriger als der Armdruck. Bei einem Quotienten von $<0,9$ liegt höchstwahrscheinlich eine PAVK vor.

Diese wird nach Fontaine in 4 Stadien eingeteilt, wobei das Stadium I asymptomatische Patienten umfasst (Tab. 1). Beinahe $20 \%$ der Patienten in der Grundversorgerpraxis haben eine PAVK $[1,2]$. Das ist alarmierend, da die Sterblichkeit innerhalb von 3 Jahren nach der Untersuchung bei PAVK-Patienten mit 10,9\% im Vergleich zu Patienten ohne PAVK mit 4,2\% deutlich erhöht ist. Auch die nicht tödlichen Ereignisse wie Herzinfarkt und Schlaganfall waren bei den Patienten mit einem verminderten ABI 3- bis 4-fach erhöht. Die Mortalität korrelierte mit dem Schweregrad der PAVK; bei ABI Werten $<0,5$ betrug sie gar $25,5 \%$ in den 3 Jahren der Untersuchung [1].

Die Atherosklerose ist ein chronisch-entzündlicher Prozess, der durch eine Aktivierung der Thrombozyten gestartet wird. Die empfohlenen medikamentösen Maßnahmen berücksichtigen dieses pathophysiologische Modell.

Die Basistherapie beinhaltet einen Thrombozytenaggregationshemmer (TAH; z.B. Acetylsalicylsäure) und ein Statin. Der TAH soll die Aktivierung der Plättchen bremsen. Die Statine werden nicht nur zur Lipidsenkung eingesetzt, sondern vor allem auch wegen der anti-inflammatorischen Wirkung zur Plaquestabilisierung. Neben Lifestyle-Beratung (Rauchstopp, Bewegung, Ernährung, usw.) ist eine medikamentöse Therapie der kardiovaskulären Risikofaktoren bei der Diagnosestellung der PAVK unverzichtbar.

Im Gegensatz zu den meisten anderen Phytotherapeutika besteht Padma ${ }^{\circledR} 28$ nicht aus dem Extrakt einer einzigen Pflanze, sondern aus einem Gemisch von 20 getrockneten Heilpflanzen. Das Präparat beinhaltet unter anderem Terpene, Flavonoide, Polysaccharide und Tannine. Es ist seit 1977 in der Schweiz registriert [3] und wird in der Gefäßmedizin auch als entzündungshemmender Wirkstoff eingesetzt. Für einige der Inhaltsstoffe von Padma 28 gibt es gute experimentelle Daten, die diese These stützen. Es liegen auch einige, allerdings ältere, kontrollierte klinische Studien vor. Eine 2006 publizierte Meta-Analyse zeigt eine gute Wirkung von Padma 28 im Hinblick auf die maximale Gehstrecke, sodass es bei allgemein guter Verträglichkeit als additive Therapie bei PAVK eingesetzt werden kann [4].

Bei starker PAVK (Stadien III und IV) ist neben der medikamentösen Therapie eine interventionelle oder chirurgische Revaskularisation unumgänglich, um eine Amputation zu vermeiden. Gefährdet sind insbesondere Diabetiker, bei denen eine diabetische Polyneuropathie (Nervenschädigung) häufig die warnenden Beschwerden maskiert. Oft sind die Arterienwände aufgrund einer Mediasklerose inkompressibel und die ABI-Werte sind nicht verwertbar. Die Indikation für eine angiologische Abklärung ist beim Diabetiker mit peripheren Läsionen deshalb sehr großzügig zu stellen.

Die interventionelle Behandlung umfasst kathetertechnische Revaskularisation mit oder ohne Stenteinlage, Rekanalisation von Verschlüssen und gefäßchirurgische Interventionen wie unter anderem Bypass, usw.

Bei Patienten mit einer Gehstreckeneinschränkung, jedoch ohne kritische Durchblutung (Stadium IIa und IIb) kann neben dem Gehtraining als additive Therapie zur Sekundärprophylaxe Padma 28 eingesetzt werden. Diese allgemein gut verträgliche Behandlung ist vor allem bei Patienten

Tab. 1. Einteilung der PAVK nach Fontaine

\begin{tabular}{ll|l}
\hline Stadium & Klinisches Bild & Therapie \\
\hline I & $\begin{array}{l}\text { nachgewiesener Verschluss oder Engstelle } \\
\text { ohne klinische Symptome }\end{array}$ & \multirow{2}{*}{$\begin{array}{l}\text { konservativ (medikamentös, } \\
\text { Lifestyle-Anpassung) }\end{array}$} \\
\cline { 1 - 2 } IIa & $\begin{array}{l}\text { Claudicatio intermittens mit einer } \\
\text { schmerzfreien Gehstrecke }>200 \mathrm{~m}\end{array}$ & $\begin{array}{l}\text { abhängig von der Morphologie } \\
\text { und den Beschwerden }\end{array}$ \\
\hline IIb & $\begin{array}{l}\text { Claudicatio intermittens mit einer } \\
\text { schmerzfreien Gehstrecke }<200 \mathrm{~m}\end{array}$ & $\begin{array}{l}\text { zusätzliche Revaskularisation } \\
\text { obligat }\end{array}$ \\
\hline III & $\begin{array}{l}\text { kritische Ischämie mit perfusionsbedingten } \\
\text { Ruheschmerzen }\end{array}$ & \\
\hline IV & zusätzlich Hautläsionen mit Gangrän &
\end{tabular}


zu empfehlen, bei denen ein interventioneller oder chirurgischer Eingriff mit sehr hohem Aufwand oder erhöhtem periinterventionellen Komplikationsrisiko verbunden ist. Ziel ist die Förderung der Kollateralisierung von meist langstreckigen Gefäßverschlüssen und mit der Verlängerung der schmerzfreien Gehstrecke die Verbesserung der Lebensqualität. Neben Lifestyle-Anpassung mit Rauchstopp, Einstellung und Behandlung kardiovaskulärer Risikofaktoren und
Gehtraining kann die Gabe von Padma 28 eine zusätzliche Unterstützung sein und einen messbaren Effekt zur Steigerung der Lebensqualität beitragen.

\section{Disclosure Statement}

Die Autoren erklären, dass kein Interessenskonflikt besteht.

\section{Literatur}

1 Diehm C, Allenberg JR, Pittrow D, Mahn M, Tepohl G, Haberl RL, Darius H, Burghaus I, Trampisch HJ; German Epidemiological Trial on Ankle Brachial Index Study Group: Mortality and vascular morbidity in older adults with asymptomatic versus symptomatic peripheral artery disease. Circulation 2009;120:2053-2061.
2 Criqui MH: Peripheral arterial disease - epidemiological aspects. Vasc Med 2001; (suppl 3):3-7.

3 Melzer J, Saller R: Das tibetische Kombinationspräparat Padma 28 bei peripherer arterieller Verschlusskrankheit. Internist Prax 2010;2:403-407.
4 Melzer J, Brignoli R, Diem C, Reichling J, Do DD, Saller R: Treating intermittent claudication with Tibetan medicine Padma 28: does it work? Atherosklerosis 2006;189:39-46.

\section{KARGER}

(ㄷ) 2013 S. Karger GmbH, Freiburg 